\title{
Neutrophilic dermatosis of the dorsal hands: a restrictive designation for an acral entity
}

\author{
Miguel Costa-Silva ${ }^{\circledR}$, Ana Pedrosa ${ }^{1,2}$, Filomena Azevedo ${ }^{1}$, Alberto Mota $^{1,2}$
}

\begin{abstract}
In 2000, Galaria et al. proposed the designation neutrophilic dermatosis of the dorsal hands (NDDH). The authors describe a case of NDDH with predominant involvement of the palmar aspect of the hands in a patient suffering from lung cancer, a possible paraneoplastic manifestation. Therefore, the term NDDH is not accurate because palmar manifestations of this dermatosis are also possible.
\end{abstract}

Keywords: neutrophilic dermatosis of the dorsal hands, Sweet's syndrome

Received: 11 October 2016 | Returned for modification: 4 November 2016 | Accepted: 10 November 2016

\section{Introduction}

In 1995, Strutton et al. (1) reported six patients with a dermatosis limited almost entirely to the dorsal aspects of the hands resembling Sweet's syndrome (SS) but differing histologically by the presence of leukocytoclastic vasculitis. In 2000, Galaria et al. (2) further described similar cases but lacking the vasculitis component; he proposed the designation neutrophilic dermatosis of the dorsal hands (NDDH). Currently, NDDH is viewed as a subset of neutrophilic dermatosis and is recognized as a localized variant of SS due to the similarities between these two entities (3).

\section{Case description}

A 63-year-old male patient was referred to our dermatology clinic due to painful violaceous bullous plaques located on the palms and dorsal aspect of the fingers of both hands with 2 weeks' evolution (Figure 1). The patient had suffered from an advanced epidermoid carcinoma of the lung for 2 years. The treatment included a cycle of gemcitabine 1 month before the clinical presentation of the lesions. At time of our observation, the patient was admitted due to an episode of hemoptysis complicated by a respiratory infection. Investigations revealed a total leukocyte count of 11.31 $\times$ 109/1 with $86.9 \%$ neutrophils and elevated C-reactive protein (176.8 mg/l). Blood cultures and pus culture from the cutaneous lesions showed no growth. Cutaneous biopsy demonstrated a dense neutrophilic infiltrate in the dermis with evidence of neu-

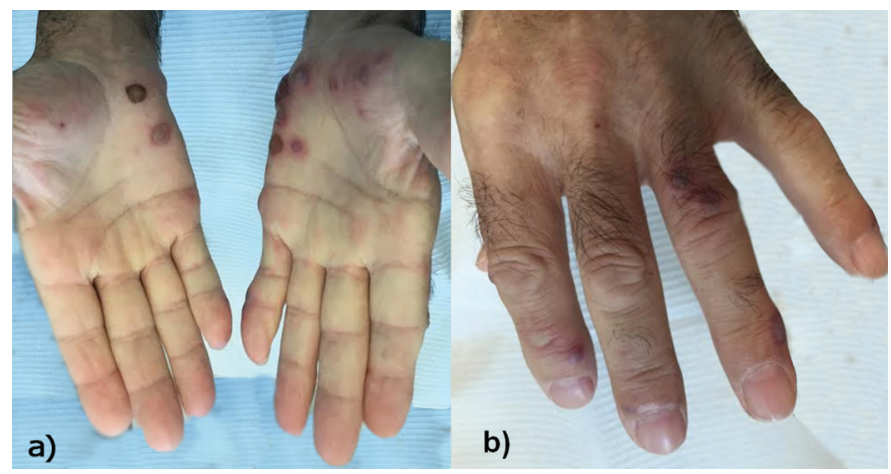

Figure 1 Bullous plaques located on the palms (A) and dorsal aspect of the fingers $(B)$ of both hands. trophilic vasculitis, consistent with the diagnosis of NDDH (Figure 2). A treatment with a course of prednisolone, $20 \mathrm{mg}$ daily, and topical betamethasone valerate resulted in a significant improvement at 1 month follow-up. The patient died 5 months after presentation due to epidermoid carcinoma of the lung with liver and brain metastases.

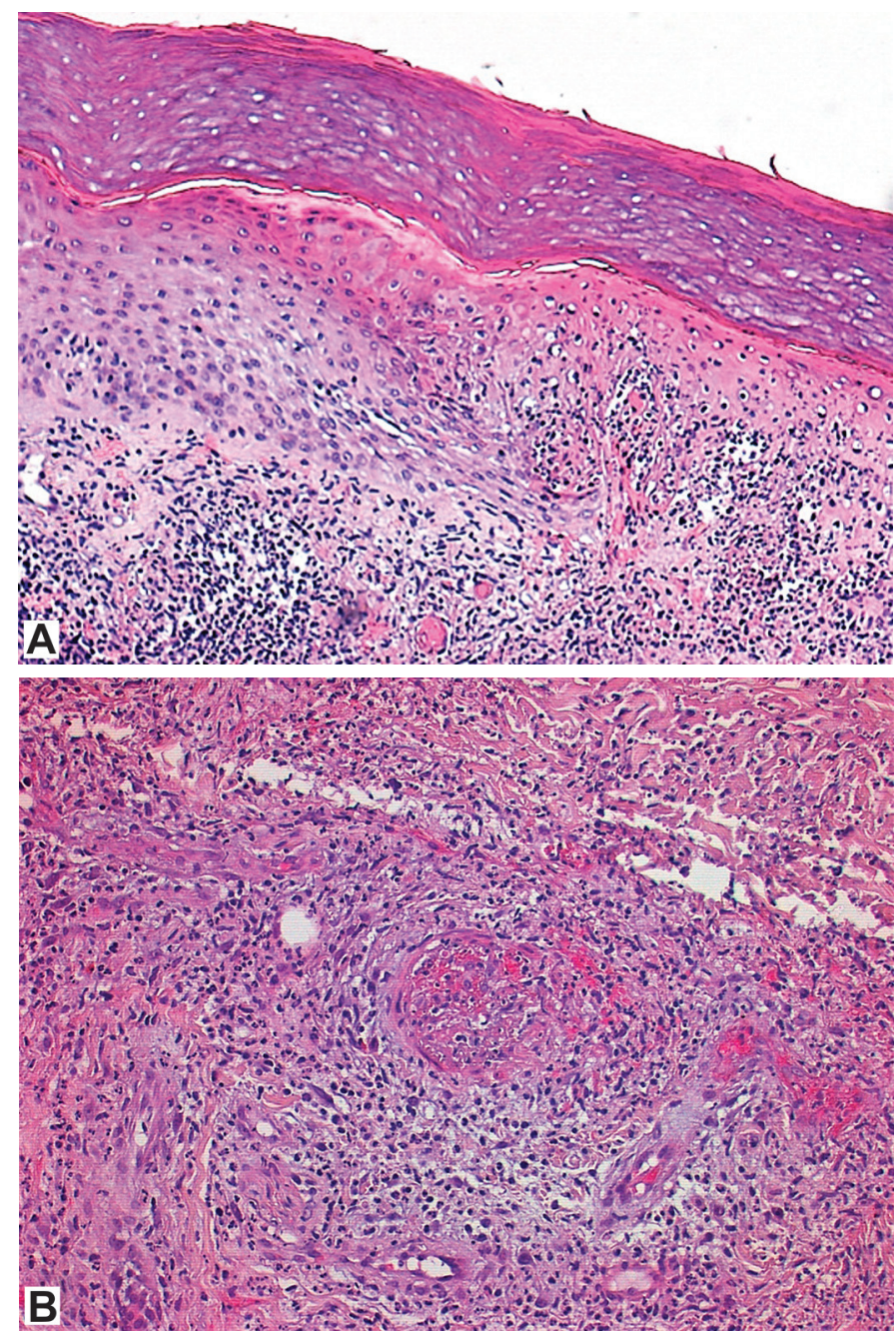

Figure 2 | A dense neutrophilic infiltrate in the dermis (A) with evidence of neutrophilic vasculitis (B) 


\section{Discussion}

NDDH and SS share similar clinical, laboratory, and histological findings (3). The most commonly associated disorders are hematological (myelodysplasia, IgA gammopathy, and B-cell lymphoma) and inflammatory (ulcerative colitis, Crohn's disease, seropositive arthritis, and sarcoidosis) $(3,4)$. There are also reports of NDDH associated to a lesser extent with infectious agents, trauma, and drugs (3). However, many cases of NDDH do not fulfill all of the SS criteria. In fact, the occurrence of constitutional signs and symptoms and the elevation of serum inflammatory markers are inconsistent in $\operatorname{NDDH}(3,4)$. Despite the fact that our patient had elevated serum inflammatory markers, he also suffered from a respiratory infection, and so it is difficult to ascribe the relative contribution to this elevation, the infection, the NDDH, or both. As with SS, most patients are treated with systemic corticosteroids (5).

Vasculitis is present in about $30 \%$ of NDDH cases, in contrast to the lack of this feature in the cases originally described as SS (5). Some authors have argued that the vascular damage in these cases is probably a secondary event related to the intensity of the neutrophilic infiltrate and do not represent true vasculitis (3-5).

Our patient predominantly had involvement of the palmar aspect of the hands in contrast to the majority of the NDDH cases reported. Nonetheless, a small number of cases involving the lateral or palmar aspect of the hands have been found in some reports

\section{References}

1. Strutton G, Weedon D, Robertson I. Pustular vasculitis of the hands. J Am Acad Dermatol. 1995;32:192-8.

2. Galaria NA, Junkins-Hopkins JM, Kligman D, James WD. Neutrophilic dermatosis of the dorsal hands: pustular vasculitis revisited. J Am Acad Dermatol. 2000; 43:870-4.

3. Cravo M, Cardoso JC, Tellechea O, Cordeiro MR, Reis JP, Figueiredo A. Neutrophilic dermatosis of the dorsal hands associated with hypopharyngeal carcinoma. Dermatol Online J. 2008;14:5.

4. Weenig RH, Bruce AJ, McEvoy MT, Gibson LE, Davis MD. Neutrophilic dermatosis of the hands: four new cases and review of the literature. Int J Dermatol. 2004;43:95-102.

5. Walling HW, Snipes CJ, Gerami P, Piette WW. The relationship between neutrophilic dermatosis of the dorsal hands and Sweet syndrome: report of 9 cases and comparison to atypical pyoderma gangrenosum. Arch Dermatol. 2006; $142: 57-63$.
$(3,4,6-8)$. This has led some authors to propose dropping dorsal from the designation of NDDH and changing it to neutrophilic dermatosis of the hands or even acral neutrophilic dermatosis (4, 6). In this respect, it is possible that NDDH as a possible paraneoplastic manifestation may in fact have a more atypical presentation, as in the case of our patient. However, an etiologic role of gemcitabine cannot be entirely ruled out in this particular case because NDDH has been associated with chemotherapy drugs (3).

\section{Conclusion}

$\mathrm{NDDH}$ is associated with potentially serious systemic conditions. This warrants awareness of NDDH in the dermatological community in order to facilitate clinical recognition and a prompt workup. The term $N D D H$ gives the false impression that this disease is strictly located on the dorsal aspect of the hands, which is not always the case, as demonstrated in this report. This may result in a more underdiagnosed disorder, and it is possible that a name change will contribute to identifying more cases.

\section{Acknowledgment}

We thank Jennifer Costa and Aurea Canellhas for their contribution to this work; in particular, the pathology investigation and photos.
6. Nofal A, Assaf M, Elakad R, Fawzy M, Nofal E. Neutrophilic dermatosis of the dorsal hands: a localized variant of Sweet's syndrome or a distinct entity? Int J Dermatol. 2015;54:e66-7.

7. DiCaudo DJ, Connolly SM. Neutrophilic dermatosis (pustular vasculitis) of the dorsal hands: a report of 7 cases and review of the literature. Arch Dermatol. 2002;138:361-5.

8. Del Pozo J, Sacristán F, Martínez W, Paradela S, Fernández-Jorge B, Fonseca E. Neutrophilic dermatosis of the hands: presentation of eight cases and review of the literature. J Dermatol. 2007;34:243-7. 